\title{
Theoretical Basis, Laboratory Evidence, and Clinical Research of Chemical Surgery of the Cornea: Cross-Linking
}

\author{
Amanda C. da Paz, ${ }^{1}$ Patrícia A. Bersanetti, ${ }^{1,2}$ Marcella Q. Salomão, ${ }^{3}$ \\ Renato Ambrósio Jr., ${ }^{1,3}$ and Paulo Schor ${ }^{1}$ \\ ${ }^{1}$ Department of Ophthalmology and Visual Sciences, Paulista School of Medicine, Federal University of São Paulo (UNIFESP), \\ São Paulo, SP, Brazil \\ ${ }^{2}$ Department of Health Informatics, Paulista School of Medicine, Federal University of São Paulo (UNIFESP), \\ 04023-062 São Paulo, SP, Brazil \\ ${ }^{3}$ Instituto de Olhos Renato Ambrósio, 20520-050 Rio de Janeiro, RJ, Brazil
}

Correspondence should be addressed to Patrícia A. Bersanetti; bersanetti@unifesp.br

Received 2 June 2014; Revised 24 July 2014; Accepted 25 July 2014; Published 18 August 2014

Academic Editor: Suphi Taneri

Copyright (C) 2014 Amanda C. da Paz et al. This is an open access article distributed under the Creative Commons Attribution License, which permits unrestricted use, distribution, and reproduction in any medium, provided the original work is properly cited.

Corneal cross-linking (CXL) is increasingly performed in ophthalmology with high success rates for progressive keratoconus and other types of ectasia. Despite being an established procedure, some molecular and clinical aspects still require additional studies. This review presents a critical analysis of some established topics and others that are still controversial. In addition, this review examines new technologies and techniques (transepithelial and ultrafast CXL), uses of corneal CXL including natural products and biomolecules as CXL promoters, and evidence for in vitro and in vivo indirect effectiveness.

\section{History}

The concept of using collagen cross-linking photochemically induced, for increasing corneal stiffness, as a conservative method to stabilize ectasia progression was first conceived in Germany in the 1990s by Theo Seiler and collaborators [1-4]. Collagen cross-linking (CXL) opened a new horizon for conscious biomechanical manipulation of the cornea [5], which uses the concept of biomechanical customization of therapeutic and refractive corneal surgery [6]. The original "Dresden cross-linking clinical protocol" involves topical anesthesia, central corneal abrasion, and application of riboflavin $0.1 \%$ with $20 \%$ dextran T-500 until stromal saturation is observed through biomicroscopy. The traditional procedure is followed by ultraviolet A (UVA) light of 365$370 \mathrm{~nm}$ at an irradiance of $3 \mathrm{~mW} / \mathrm{cm}^{2}$, which corresponds to a dose of $5.4 \mathrm{~J} / \mathrm{cm}^{2}$ for $30 \mathrm{~min}[3]$.

The photopolymerization effect on corneal collagen results from the reaction of the photosensitizer agent riboflavin and UVA light $(370 \mathrm{~nm})$, which is the absorptive peak of riboflavin. This reaction generates reactive oxygen species that can react with various molecules and subsequently induce chemical covalent bonds that bridge the amino groups of collagen fibrils [7]. Hayes et al. (2013) demonstrated riboflavin/UVA-induced cross-links at the surface of the collagen fibrils and within the proteoglycan (PG) rich coating surrounding them [8]. In another study, Zhang et al. (2011) reported that riboflavin/UVA treatment causes beyond CXL among collagen molecules and among PG core proteins, as well as limited linkages between collagen and PG such as mimecan, decorin, keratocan, and lumican [9].

\section{Other Approaches}

2.1. Natural Cross-Linking. Human collagen undergoes progressive changes including a decrease in solubility, elasticity, and permeability, as well as an increase in thermal stability and resistance to enzymatic digestion with aging. The precise chemical changes of these transformations are unknown. However, an in vitro study has suggested that these physical changes involve progressive CXL among collagen molecules 
[10]. A detailed study of the collagen fibrils in normal human corneas showed a small but significant age-related increase in collagen fibrils for diameter, intermolecular spacing, and elongation [11]. Expansion of the collagen intermolecular spacing suggests molecules other than collagen are deposited between the fibrils during aging, which subsequently push the collagen molecules further apart. This is consistent with a recent study that demonstrated glycation-induced expansion of intermolecular spacing and subsequent CXL of molecules with age [10]. Considering the isolated ultrastructural dimensions of collagen fibrils, one would expect a tendency toward biomechanical strengthening of the cornea during aging [12].

Hyperglycemia was shown to influence corneal biomechanical properties by inducing stromal collagen CXL through glycosylation and lysyl oxidase (LOX) enzymatic activity [13]. People with diabetes mellitus have increased central corneal thickness, corneal hysteresis, and a corneal resistance factor, possibly reflecting a greater stiffness of diabetic corneas [14].

2.2. Biomolecules and Natural Products. Several studies have demonstrated several molecules that might promote collagen cross-link. Natural products such as genipin $[15,16]$ and proanthocyanidins (PAs) [17] can form cross-links between collagen fibrils. Avila et al. (2012) demonstrated in an ex vivo study that corneal CXL with genipin was similar to the UV traditional procedure, with minimal toxicity to endothelial cells [16]. PAs are natural products with polyphenolic structures that have the potential to give rise to stable hydrogen bonded structures and generate nonbiodegradable collagen matrices. Han et al. (2003) demonstrated the feasibility of using PAs from grape seeds to cross-link collagenous materials [17].

Biomolecules, such as the leucine-rich proteoglycans (e.g., decorin, lumican, and keratocan), regulate the orderly assembly of extracellular matrices, corneal transparency, tensile strength of skin and tendons, viscoelasticity of blood vessels, and tumor cell proliferation. Experiments in vitro showed that SLRPs interact with collagen through specific binding sites and delay formation of collagen fibrils. To modulate cornea collagen fibrillogenesis decorin binds to collagen types I, II, III, VI, and XIV [18, 19].

\section{In Vitro Effectiveness Evidences}

3.1. Increase in Collagen Fiber Diameter. Riboflavin/UVAinduced collagen CXL increases the corneal collagen fiber diameter, which was more pronounced in the anterior portion of the stroma of the rabbit cornea as observed on transmission electron microscopy [23].

3.2. Resistance to Enzymatic Digestion. The stabilizing biochemical effect of CXL can be explained by changes in the tertiary structure of collagen fibrils induced by CXL preventing access of the proteolytic enzymes to their specific cleavage sites by steric hindrance. In porcine corneas cross-linked with riboflavin/UVA, CXL causes an impressive doubling in the time following pepsin, trypsin, and collagenase digestion, particularly in the anterior half of the cornea [22].

3.3. Modulus of Elasticity (Young's Modulus). Many published studies report an increase in cornea stiffness after collagen CXL. Wollensak et al. (2003) found a significant increase in biomechanical rigidity by a factor of 4.5 in human corneas following riboflavin/UVA-induced collagen CXL, which was indicated by an increase in Young's modulus. The increase in biomechanical stiffness in porcine eyes was also significant by a factor of 1.8 [20]. In another study, Wollensak and Iomdina (2009) found a highly significant increase in corneal stiffness after CXL treatment of rabbit corneas with an impressive durability over time, as demonstrated by a $78.4 \%-87.4 \%$ (by a factor of 1.6) increase in Young's modulus by and a 69.7\%$106.0 \%$ increase in ultimate stress over the entire 8-month follow-up [21]. Some limitations of this method are that the strip specimens originated from a curved sample, the corneal structure is disrupted because the lamellae are cut, and several crucial constraints are ignored (e.g., real pachymetry and meridional differences) [31].

3.4. Atomic Force Microscopy. Atomic force microscopy (AFM) has a shaping probe tip that can scan the sample surface at an atomic distance. By monitoring the interaction force between the tip and the sample surface, this instrument can create topographical images of the sample surface at high resolutions [32]. When the probes approach the sample surface, tiny interaction forces, such as Van der Waals and electrostatic forces, occur between the probe and sample. The resulting cantilever is recorded by measuring the displacement of a laser beam reflected from the backside of the cantilever. AFM can be applied to identify the collagen bundles and to determine their diameters [33]. This technique provides quantitative information on the surface morphology of the collagen fibrils at a high resolution [32]. Yamamoto et al. (2002) clearly obtained surface topographic images of human corneal and scleral collagen fibrils using AFM [32]. Further AFM studies are important to examine cross-link induced modification in corneal collagen fibrils. Seifert et al. (2014) developed a method that allows for atomic force microscopy-based measurements of gradients of Young's modulus in soft tissues. In the abovementioned study, the authors demonstrated the depth-dependent distribution of the stiffening effect caused by riboflavin/UV CXL in porcine corneas [34].

3.5. X-Ray Scattering. X-ray scattering is a specialized technique that provides structural information about the constituent collagen in the corneal stroma. The wide-angle equatorial scattering pattern produced from the lateral packing of molecules within the stromal collagen fibrils can be used to determine the intermolecular spacing within the fibrils, as well as the arrangement and distribution of fibrillar collagen in the intact cornea $[35,36]$. X-ray scattering is a unique method for measuring the lateral space between individual fibril-forming collagen molecules at less than a $1 \mathrm{~mm}$ resolution. This space is influenced by both the fibril 
hydration and the extent of molecular CXL [35]. Studies of corneal collagen organization in keratoconus (KC) suggest that the mechanism of tissue thinning in this disease involves fibrillar or lamellar collagen slippage, decreased lamellar interweaving [35,37], and distortion of the orthogonal matrix [37]. The authors of study proposed that development of interventional cross-linking strategies may limit collagen slippage and should be beneficial for delaying the progression of keratoconus [35, 37]. In another study that analyzed CXL in human corneas using X-ray scattering, Hayes et al. (2011) concluded that UVA/riboflavin induced cross-links do not have a measurable effect on the axial stagger or the tilt of collagen molecules within the fibrils when analyzed using Xray scattering method [36].

3.6. Second Harmonic Generation Microscopy. Second harmonic generation (SHG) microscopy has been used extensively in medicine and biology to obtain images of highly ordered structures, such as collagen fibers, microtubulin, and skeletal muscle, with high resolution and contrast. This nonlinear optical microscopy results from a coherent secondorder nonlinear scattering wherein a noncentrosymmetric structure emits light at half the wavelength of the incident (pump) optical field. Collagen fibers, being intrinsically noncentrosymmetric, emit SHG and thus produce high-contrast images without the need for staining [38].

Collagen fibrils are aligned uniformly in the corneal stroma and are therefore believed to be responsible for SHG from the cornea. SHG imaging has thus allowed visualization of collagen organization and can be processed to generate three-dimensional reconstructions of collagen structure [39].

In 12 of 13 human keratoconic corneal samples obtained after penetrating keratoplasty for KC, SHG could detect differences in the organizational pattern of lamellae, including a marked loss or decrease in anterior lamellae interweaving and lamellae that inserted into Bowman's layers [40].

Analysis of porcine corneas with and without riboflavin/UVA CXL treatment using SHG showed that stromal collagen fibrils in untreated corneas had a more regular, linear, and parallel orientation. However, treated corneas had wavy stromal collagen fibrils [41].

\section{In Vivo Indirect Effective Evidences}

4.1. Visual Acuity. The primary goal of CXL is to improve the biomechanical rigidity of corneal collagen to stop ectasia progression $[1,2]$. In the first published clinical trial, Wollensak et al. (2003) reported stability after CXL treatment of the eyes of 19 patients with progressive $\mathrm{KC}$ and with a mean follow-up of 20 months (from 3 to 33 months) [3]. In this series, visual acuity (VA) slightly improved in 15 eyes (65\%). The improved uncorrected visual acuity (UCVA) recorded during the follow-up is partially explained by the sphere and spherical equivalent reduction. However, these data also may be related to a progressive reduction of the mean $\mathrm{K}$ power. Furthermore, the increased best spectacle-corrected visual acuity (BSCVA) may be linked to a reduction in the difference between superior and inferior corneal hemimeridians (flattest versus steeper), expressed by the improvement in corneal symmetry indexes. Moreover, an increased BSCVA may be sustained by the statistically significant early reduction in coma aberration [42].

4.2. Keratometry. In the first published clinical trial [3], there was a variable disease regression observed in 16 cases (70\%) by a reduction of the maximal keratometry readings and refractive error [3]. Similar results were observed in other studies examining CXL for KC [43-50] and keratectasia [48, 51-54]. Corneal reshaping [55] appears to be a more reliable expression of CXL induced clinical and topographic changes. Mean clinical and topographic improvements were recorded from the end of the third postoperative month and continued thereafter, reaching reliable stability in 24 months [46]. In addition, Koller et al. (2009) found KMax to be an important prognostic variable, which was associated with a significant reduction in complications when excluding cases with a KMax higher than 58D [56]. A higher chance of ectasia regression, observed by flattening, was more likely if KMax was higher than 54D [57].

4.3. Biomicroscopy. A stromal demarcation line, biomicroscopically detectable as early as 2 weeks after CXL treatment, was described by Seiler and Hafezi (2006) as the first clinical evidence of a physical effect of CXL on corneal tissue [58]. The demarcation line does not refer to biomechanical properties but represents the transition between cross-linked anterior corneal stroma, with modified refractive and reflection properties, and the untreated posterior corneal stroma [58]. Caporossi et al. (2010) found stromal edema, clinically detectable by slit-lamp examination in $70 \%$ of patients, occurred in the first 30 postoperative days. Temporary haze occurred in $9.8 \%$ of cases, 14 cases in the first 3 months, and 2 cases after 6 months but disappeared progressively after topical preservative-free steroid therapy [46].

4.4. Scheimpflug Photography and Optical Coherence Tomography. The stromal demarcation line is also observed via Scheimpflug photography [59-62] and optical coherence tomography (OCT) [46, 63]. Visante OCT scans show a higher reflectivity (hyperdensity) of this line, and after 6 months, stromal reflectivity becomes more homogeneous, reducing the visibility of the line in some eyes much more than in others [46].

4.5. Pachymetry. The pachymetric map provides the thinnest point data, which is critical for ensuring the safety parameters for the endothelium [64]. The thickness map also should be important for monitoring results after CXL. Corneal thinning has been documented in the early CXL postoperative course, with a gradual return on corneal thickness toward preoperative values within the first year after CXL $[45,46,62,65]$.

4.6. Ocular Response Analyzer. Until the launching of the ocular response analyzer (ORA) (Reichert Inc., Depew, NY) in 2005 [66], corneal biomechanical studies were limited to 
laboratory in vitro studies and virtual mathematical corneal finite element models $[67,68]$. ORA is a modified noncontact tonometer (NCT) that was designed to provide a more accurate measurement of IOP through an understanding of compensation for corneal properties [66].

During an ORA measurement, a precisely metered air pulse is delivered to the eye, causing the cornea to move inward, past a first applanation (flattening), and into a slight concavity. Milliseconds after the first applanation, the air pump generating the air pulse is shut down and the pressure applied to the eye decreases in an inverse-time symmetrical fashion. As the pressure decreases, the cornea passes through a second applanated state while returning from concavity to its normal convex curvature [66].

An electrooptical collimation detector system monitors the corneal curvature in the central $3.0 \mathrm{~mm}$ diameter throughout the 20-millisecond measurement. A filtered (smoothed) version of the detector signal defines 2 precise applanation times corresponding to 2 well-defined peaks produced by inward and outward applanation events. Two corresponding pressures of an internal air supply plenum are determined from the applanation times derived from the detector applanation peaks [66].

The system registers the independent applanation pressures during the ingoing (P1) and outgoing (P2) phases. The difference between the 2 pressures is called corneal hysteresis $(\mathrm{CH})[69,70]$. Corneal resistance factor $(\mathrm{CRF})$ is also calculated from P1 and P2 with an optimized function designed to augment the correlation with thickness in a normal population $[66,70]$. $\mathrm{CH}$ and $\mathrm{CRF}$ were significantly lower in keratoconus, but $\mathrm{CH}$ and $\mathrm{CRF}$ were unchanged after CXL [71-73]. Hysteresis is a viscoelastic property of the cornea that is not directly related to stiffness [74]. A new set of parameters derived from the waveform ORA signal that monitors the deformation response of the cornea during an ORA measurement has been reported [72-76]. These parameters had a better diagnostic performance for keratoconus $[75,76]$ and improved after CXL $[74,76]$.

4.7. Corvis. Corvis has an ergonomic design. The patient is comfortably positioned with proper placement of the chin and forehead and then asked to focus on a central red LED. A frontal view camera is mounted with a keratometer-type projection system for focusing and aligning the corneal apex. The examination is programmed for automatic release when alignment is achieved with the first Purkinje reflex of the cornea [77].

This equipment is a NCT system integrated with an ultrahigh speed (UHS) Scheimpflug camera that was introduced by Ambrósio Jr et al. (2013) [77]. The CorVis ST (Scheimpflug Technology) records 4,330 frames per second, with a Scheimpflug camera that covers $8 \mathrm{~mm}$ horizontally, to monitor the corneal response to a fixed profile air pulse with a maximal internal pump pressure of $25 \mathrm{kPa}$. The addition of an UHS Scheimpflug camera allows dynamic inspection of the actual deformation process that provides further details for biomechanical characterization of the cornea.

The recording starts with the cornea at the natural convex shape. The air puff forces the cornea inward (ingoing phase) through applanation (first or ingoing applanation) into a concavity phase until it achieves the highest concavity (HC). Thereafter, the cornea undergoes a second applanation before achieving its natural shape [77]. The parameters derived from the corneal response such as corneal speed during deformation, corneal applanation length, deformation amplitude (greatest displacement of the apex at the point of $\mathrm{HC}$ ), and radius of curvature at $\mathrm{HC}$ are important measures of corneal viscoelastic properties and stiffness. Such parameters are useful for the diagnosis of ectasia [75] and assessing CXL results.

In an ancillary study conducted at the Ohio State University in an industry-sponsored FDA trial of corneal collagen CXL, subjects were evaluated biomechanically using the CorVis ST before and after the procedure. Preliminary analysis at 1-month postprocedure was performed with $11 \mathrm{ker}-$ atoconic subjects randomly selected for treatment, compared with 8 keratoconic subjects randomly selected for the sham group. A significant difference $(P<0.0014)$ was found in the radius of curvature at $\mathrm{HC}$ in subjects who received treatment, which is consistent with increased stiffness. Subjects in the sham group showed no difference $(P=0.6981)$ at 1 month [77].

4.8. Confocal Microscopy. In vivo confocal analysis showed disappearance of keratocytes in the anterior midstroma to a depth of $340 \mu \mathrm{m}$ [55] and a clear vertical transition area between the edematous hyporeflective stroma with apoptotic bodies and normoreflective deep stroma. After 6 months, the reflectivity of the anterior midstroma was inverted (hyper) compared with initial postoperative reflective previously demonstrated [55]. Changes in the stromal reflectivity after the sixth month are an important indirect (confocal) sign of corneal CXL [55]. In general, after the third month, there is new collagen synthesis meditated by repopulating keratocytes and lamellar compaction, expressed by the hyperreflectiveness of the extracellular matrix, combined with newly formed collagen fibers identified with in vivo confocal scans [55, 78]. In addition to this finding, nerve plexus degeneration was noted up to 6 months postoperatively following CXL [79].

Confocal microscopy demonstrated numerous hyperreflective spherical structures more abundantly in the anterior stroma, and they were visible up to a depth of $300 \mu \mathrm{m}$ after CXL [80]. It is not clear what these structures represent; however, they may represent damaged keratocytes or nuclear and cellular fragments. The stroma had a spiculated appearance and extended to a depth at $300 \mu \mathrm{m}$ that could be secondary to changes in stromal hydration [80].

The increase of collagen fiber diameter could partly explain the increased scattering of the collagen fibers creating a net-like formation observed at the first and third months after CXL [81]. In addition, revelation of the otherwise unseen collagen fibers in the confocal microscopy images also suggest alterations of the normal collagen fiber formation that is responsible for the transparency of the cornea in normal conditions. This may also have implications on the vision function and contrast sensitivity [81]. 
TABLE 1: In vitro and in vivo evidences of corneal cross-linking protocols.

\begin{tabular}{lll}
\hline Protocol & In vitro & In vivo \\
\hline Epi-off CXL (Dresden Protocol) & $\begin{array}{l}\text { Increased Young's modulus [20, 21], resistance to } \\
\text { enzymatic degradation [22], and collagen fiber } \\
\text { diameter [23] }\end{array}$ & $\begin{array}{l}\text { Improvement in VA, K reading, refraction, and } \\
\text { halt of ectasia progression }\end{array}$ \\
\hline Eiboflavin penetration requires more time than with \\
epi-off techniques \\
$\begin{array}{ll}\text { Epithelium permeabilization can be achieved with } \\
\text { molecules as cyclodextrins [24] and benzalkonium } \\
\text { chloride in association with NaCl [25] }\end{array}$ & $\begin{array}{l}\text { Improvement in VA and topographic findings } \\
\text { Halt of ectasia progression } \\
\text { There is a lot of controversy about results of } \\
\text { this technique [26] }\end{array}$ \\
\hline Ultrafast CXL & Young's modulus similar to traditional CXL [27] & $\begin{array}{l}\text { Equivalent in VA, refraction and pentacam } \\
\text { parameters [28, 29], and OCT imaging [29] }\end{array}$ \\
\hline Athens protocol & No data available & $\begin{array}{l}\text { Superiorly with a better BSCVA, mean K } \\
\text { reduction, spherical equivalent, and corneal } \\
\text { haza score [30] }\end{array}$ \\
\hline
\end{tabular}

\section{Another Crosslinking Protocols}

5.1. Transepithelial Cross-Linking (Epithelial Damage versus Amphiphilic Molecules). Analysis of the light transmission spectra of porcine corneas following riboflavin/UVA corneal CXL treatment suggests a need for completely removing the epithelium to allow adequate and homogeneous penetration of riboflavin into the stroma [82]. A grid pattern of full thickness epithelial debridement appears to allow some riboflavin stromal penetration; however, this was less significant compared with that observed after complete central epithelium removal [82]. An application of $20 \%$ alcohol in the presence of an intact epithelium is not sufficient to allow adequate riboflavin penetration into the corneal stroma [82]. A riboflavin complex with ethylenediaminetetraacetic acid (EDTA) and trometamol was used for transepithelial CXL after superficial scraping. However, the uptake was considerably less than in corneas with epithelium removed [83]. Pharmacological permeabilization of epithelium was achieved by applying cyclodextrins that enhance riboflavin solubility in water and to improve its permeability through bovine corneas [24]. Raiskup et al. (2012) showed that a riboflavin solution without dextran, but including $0.01 \%$ benzalkonium chloride and $0.44 \% \mathrm{NaCl}$ promoted the permeability through the epithelium, resulting in a sufficient concentration of riboflavin in the stroma [25]. Recently, Bottos et al. (2013) described riboflavin nanoemulsions that could penetrate the corneal epithelium. A greater stromal concentration was detected after 240 min when compared with corneas submitted to the standard protocol [84]. Bikbova and Bikbov (2014) showed the effectiveness of the impregnation of riboflavin $0.1 \%$ in eyes of 19 patients by iontophoresis in transepithelial collagen CXL with a decrease in the average keratometry 1 year after the procedure [85].

5.2. Athens Protocol. Kanellopoulus et al. (2009) studied topography-guided PRK at least 6 months following CXL and topography PRK followed immediately by CXL in a single procedure in adults with advancing $\mathrm{KC}$ to stabilize ectasia and rehabilitate vision (with topography-guided PRK) [30]. The simultaneous procedure appeared to be superior to sequential treatments in rehabilitation of keratoconus with minimal haze formation, and in addition to a reduction in the patient's time away from work. Perhaps CXL will have a wider application as prophylaxis in laser refractive surgery [30]. In another study, the same author found potentially promising results with the same-day and simultaneous topographyguided PRK and collagen CXL as a therapeutic intervention in highly irregular corneas with progressive corneal ectasia after LASIK [86].

5.3. Ultrafast Cross-Linking. According to the Bunsen and Roscoe (1862) law, the effect of a photochemical or photobiological reaction is directly proportional to the total irradiation dose, irrespective of the time span over which the dose is administered [87]. Schumacher et al. (2011) found an increase in Young's modulus statistically equivalent in the group of porcine corneas treated with illumination intensity of $10 \mathrm{~mW} / \mathrm{cm}^{2}$ and 3 times shorter illumination time of $9 \mathrm{~min}$ compared with a group with an intensity of $3 \mathrm{~mW} / \mathrm{cm}^{2}$ that required an illumination time of $30 \mathrm{~min}$ [27]. High fluence and UV light used with shorter exposure appears to be safe and effective in stabilizing keratoconus, and this technique appears to be similar but more comfortable for patients [28].

In Table 1 are showed in vitro and in vivo evidences of Dresden protocol and the new approaches of CXL.

\section{Conclusions}

Clinical assessment of biomechanical properties represents an area of active research. Novel nondestructive methodologies have been described, including radial shearing speckle pattern interferometry [88, 89], Brillouin optical microscopy [90], and other forms of dynamic corneal imaging [91, 92]. These approaches may soon be developed into commercially available instruments.

CXL has revolutionized the treatment of ectatic diseases. However, considering the goal of the procedure is to stiffen corneal tissue, thereby stabilizing ectasia progression, characterization of the cornea should go beyond shape analysis into biomechanical assessment. Such characterization is critical 
for enabling conscious optimization and further improvements in CXL techniques. Such advances should significantly affect the indication, planning, and postoperative evaluation of ectasia treatments.

\section{Conflict of Interests}

Amanda C. da Paz, Patrícia A. Bersanetti, and Marcella Q. Salomão declare that they have no conflict of interests; Renato Ambrósio Jr.: consultant for Alcon and Oculus Optikgeräte GmbH; P. Schor: patent PI1001009-2, deposited on 03/26/2010, at Brazilian National Institute of Industrial Property (INPI-http://www.inpi.gov.br).

\section{Acknowledgments}

The authors acknowledge the financial support of FAPESP (Process 2012/07343-8 and 2013/20857-3) and CNPq.

\section{References}

[1] E. Spoerl, M. Huhle, and T. Seiler, "Induction of cross-links in corneal tissue," Experimental Eye Research, vol. 66, no. 1, pp. 97103, 1998.

[2] E. Spoerl and T. Seiler, "Techniques for stiffening the cornea," Journal of Refractive Surgery, vol. 15, no. 6, pp. 711-713, 1999.

[3] G. Wollensak, E. Spoerl, and T. Seiler, "Riboflavin/ultraviolet-Ainduced collagen crosslinking for the treatment of keratoconus," American Journal of Ophthalmology, vol. 135, no. 5, pp. 620-627, 2003.

[4] G. Wollensak, "Crosslinking treatment of progressive keratoconus: new hope," Current Opinion in Ophthalmology, vol. 17, no. 4, pp. 356-360, 2006.

[5] R. R. Krueger, "Biomechanical manipulation: The next frontier in corneal refractive surgery," Journal of Refractive Surgery, vol. 25, no. 10, pp. 837-840, 2009.

[6] C. Roberts, "Biomechanical customization: the next generation of laser refractive surgery," Journal of Cataract and Refractive Surgery, vol. 31, no. 1, pp. 2-5, 2005.

[7] C. M. Krishna, S. Uppuluri, P. Riesz, J. S. Zigler Jr., and D. Balasubramanian, "A study of the photodynamic efficiencies of some eye lens constituents," Photochemistry and Photobiology, vol. 54, no. 1, pp. 51-58, 1991.

[8] S. Hayes, C. S. Kamma-Lorger, C. Boote et al., "The effect of riboflavin/UVA collagen cross-linking therapy on the structure and hydrodynamic behaviour of the ungulate and rabbit corneal stroma," PLoS ONE, vol. 8, no. 1, Article ID e52860, 2013.

[9] Y. Zhang, A. H. Conrad, and G. W. Conrad, "Effects of ultraviolet-A and riboflavin on the interaction of collagen and proteoglycans during corneal cross-linking," Journal of Biological Chemistry, vol. 286, no. 15, pp. 13011-13022, 2011.

[10] N. S. Malik, S. J. Moss, N. Ahmed, A. J. Furth, R. S. Wall, and K. M. Meek, "Ageing of the human corneal stroma: structural and biochemical changes," Biochimica et Biophysica ActaMolecular Basis of Disease, vol. 1138, no. 3, pp. 222-228, 1992.

[11] A. Daxer, K. Misof, B. Grabner, A. Ettl, and P. Fratzl, "Collagen fibrils in the human corneal stroma: structure and aging," Investigative Ophthalmology and Visual Science, vol. 39, no. 3, pp. 644-648, 1998.
[12] A. Daxer, "Age-related corneal biomechanical changes," Journal of Cataract and Refractive Surgery, vol. 34, no. 5, pp. 715-716, 2008.

[13] C. Sady, S. Khosrof, and R. Nagaraj, "Advanced Maillard reaction and crosslinking of corneal collagen in diabetes," Biochemical and Biophysical Research Communications, vol. 214, no. 3, pp. 793-797, 1995.

[14] Y. Goldich, Y. Barkana, Y. Gerber et al., "Effect of diabetes mellitus on biomechanical parameters of the cornea," Journal of Cataract and Refractive Surgery, vol. 35, no. 4, pp. 715-719, 2009.

[15] M. Y. Avila and J. L. Navia, "Effect of genipin collagen crosslinking on porcine corneas," Journal of Cataract and Refractive Surgery, vol. 36, no. 4, pp. 659-664, 2010.

[16] M. Y. Avila, V. A. Gerena, and J. L. Navia, "Corneal crosslinking with genipin, comparison with UV-riboflavin in ex-vivo model," Molecular Vision, vol. 18, pp. 1068-1073, 2012.

[17] B. Han, J. Jaurequi, B. W. Tang, and M. E. Nimni, "Proanthocyanidin: a natural crosslinking reagent for stabilizing collagen matrices," Journal of Biomedical Materials Research A, vol. 65, no. 1, pp. 118-124, 2003.

[18] S. Kalamajski and Å. Oldberg, "The role of small leucine-rich proteoglycans in collagen fibrillogenesis," Matrix Biology, vol. 29, no. 4, pp. 248-253, 2010.

[19] D. R. Keene, J. D. San Antonio, R. Mayne et al., "Decorin binds near the C terminus of type I collagen," Journal of Biological Chemistry, vol. 275, no. 29, pp. 21801-21804, 2000.

[20] G. Wollensak, E. Spoerl, and T. Seiler, "Stress-strain measurements of human and porcine corneas after riboflavinultraviolet-A-induced cross-linking," Journal of Cataract and Refractive Surgery, vol. 29, no. 9, pp. 1780-1785, 2003.

[21] G. Wollensak and E. Iomdina, "Long-term biomechanical properties of rabbit cornea after photodynamic collagen crosslinking," Acta Ophthalmologica, vol. 87, no. 1, pp. 48-51, 2009.

[22] E. Spoerl, G. Wollensak, and T. Seiler, "Increased resistance of crosslinked cornea against enzymatic digestion," Current Eye Research, vol. 29, no. 1, pp. 35-40, 2004.

[23] G. Wollensak, M. Wilsch, E. Spoerl, and T. Seiler, "Collagen fiber diameter in the rabbit cornea after collagen crosslinking by riboflavin/UVA," Cornea, vol. 23, no. 5, pp. 503-507, 2004.

[24] P. W. J. Morrison, C. J. Connon, and V. V. Khutoryanskiy, "Cyclodextrin-mediated enhancement of riboflavin solubility and corneal permeability," Molecular Pharmaceutics, vol. 10, no. 2, pp. 756-762, 2013.

[25] F. Raiskup, R. Pinelli, and E. Spoerl, "Riboflavin osmolar modification for transepithelial corneal cross-linking," Current Eye Research, vol. 37, no. 3, pp. 234-238, 2012.

[26] M. Filippello, E. Stagni, and D. O'Brart, "Transepithelial corneal collagen crosslinking: bilateral study," Journal of Cataract and Refractive Surgery, vol. 38, no. 2, pp. 283-291, 2012.

[27] S. Schumacher, L. Oeftiger, and M. Mrochen, "Equivalence of biomechanical changes induced by rapid and standard corneal cross-linking, using riboflavin and ultraviolet radiation," Investigative Ophthalmology and Visual Science, vol. 52, no. 12, pp. 9048-9052, 2011.

[28] A. J. Kanellopoulos, "Long term results of a prospective randomized bilateral eye comparison trial of higher fluence, shorter duration ultraviolet $\mathrm{A}$ radiation, and riboflavin collagen cross linking for progressive keratoconus," Clinical Ophthalmology, vol. 6, no. 1, pp. 97-101, 2012.

[29] A. J. Kanellopoulos, "Long-term safety and efficacy follow-up of prophylactic higher fluence collagen cross-linking in high 
myopic laser-assisted in situ keratomileusis," Clinical Ophthalmology, vol. 6, no. 1, pp. 1125-1130, 2012.

[30] A. J. Kanellopoulos, "Comparison of sequential vs same-day simultaneous collagen cross-linking and topography-guided PRK for treatment of keratoconus," Journal of Refractive Surgery, vol. 25, no. 9, pp. S812-S818, 2009.

[31] S. Kling, L. Remon, A. Pérez-Escudero, J. Merayo-Lloves, and S. Marcos, "Corneal biomechanical changes after collagen crosslinking from porcine eye inflation experiments," Investigative Ophthalmology and Visual Science, vol. 51, no. 8, pp. 3961-3968, 2010.

[32] S. Yamamoto, J. Hitomi, S. Sawaguchi, H. Abe, M. Shigeno, and T. Ushiki, "Observation of human corneal and scleral collagen fibrils by atomic force microscopy," Japanese Journal of Ophthalmology, vol. 46, no. 5, pp. 496-501, 2002.

[33] S. Thalhammer, W. M. Heckl, A. Zink, and A. G. Nerlich, "Atomic force microscopy for high resolution imaging of collagen fibrils- A new technique to investigate collagen structure in historic bone tissues," Journal of Archaeological Science, vol. 28, no. 10, pp. 1061-1068, 2001.

[34] J. Seifert, C. M. Hammer, J. Rheinlaender et al., "Distribution of Young's modulus in porcine corneas after riboflavin/UVAinduced collagen cross-linking as measured by atomic force microscopy," PLoS ONE, vol. 9, no. 1, Article ID e88186, 2014.

[35] S. Hayes, C. Boote, S. J. Tuft, A. J. Quantock, and K. M. Meek, "A study of corneal thickness, shape and collagen organisation in keratoconus using videokeratography and X-ray scattering techniques," Experimental Eye Research, vol. 84, no. 3, pp. 423434, 2007.

[36] S. Hayes, C. Boote, C. S. Kamma-Lorger et al., "Riboflavin/UVA collagen cross-linking-induced changes in normal and keratoconus corneal stroma," PLOS ONE, vol. 6, no. 8, Article ID e22405, 2011.

[37] K. M. Meek, S. J. Tuft, Y. Huang et al., "Changes in collagen orientation and distribution in keratoconus corneas," Investigative Ophthalmology \& Visual Science, vol. 46, no. 6, pp. 1948-1956, 2005.

[38] R. A. R. Rao, M. R. Menta, and K. C. Toussaint Jr., "Fourier transform-second-harmonic generation imaging of biological tissues," Optics Express, vol. 17, no. 17, pp. 14534-14542, 2009.

[39] N. Morishige, N. Yamada, S. Teranishi, T. Chikama, T. Nishida, and A. Takahara, "Detection of subepithelial fibrosis associated with corneal stromal edema by second harmonic generation imaging microscopy," Investigative Ophthalmology and Visual Science, vol. 50, no. 7, pp. 3145-3150, 2009.

[40] N. Morishige, A. J. Wahlert, M. C. Kenney et al., "Secondharmonic imaging microscopy of normal human and keratoconus cornea," Investigative Ophthalmology and Visual Science, vol. 48, no. 3, pp. 1087-1094, 2007.

[41] H. Tan, Y. Chang, W. Lo et al., "Characterizing the morphologic changes in collagen crosslinked-treated corneas by Fourier transform-second harmonic generation imaging," Journal of Cataract and Refractive Surgery, vol. 39, no. 5, pp. 779-788, 2013.

[42] A. Caporossi, S. Baiocchi, C. Mazzotta, C. Traversi, and T. Caporossi, "Parasurgical therapy for keratoconus by riboflavinultraviolet type A rays induced cross-linking of corneal collagen.: preliminary refractive results in an Italian study," Journal of Cataract and Refractive Surgery, vol. 32, no. 5, pp. 837-845, 2006.

[43] F. Raiskup-Wolf, A. Hoyer, E. Spoerl, and L. E. Pillunat, "Collagen crosslinking with riboflavin and ultraviolet-A light in keratoconus: long-term results," Journal of Cataract and Refractive Surgery, vol. 34, no. 5, pp. 796-801, 2008.

[44] C. Wittig-Silva, M. Whiting, E. Lamoureux, R. G. Lindsay, L. J. Sullivan, and G. R. Snibson, "A randomized controlled trial of corneal collagen cross-linking in progressive keratoconus: preliminary results," Journal of Refractive Surgery, vol. 24, supplement 7, pp. S720-S725, 2008.

[45] P. Vinciguerra, E. Albè, S. Trazza et al., "Refractive, topographic, tomographic, and aberrometric analysis of keratoconic eyes undergoing corneal cross-linking," Ophthalmology, vol. 116, no. 3, pp. 369-378, 2009.

[46] A. Caporossi, C. Mazzotta, S. Baiocchi, and T. Caporossi, "Longterm results of riboflavin ultraviolet a corneal collagen crosslinking for keratoconus in Italy: the Siena eye cross study," The American Journal of Ophthalmology, vol. 149, no. 4, pp. 585-593, 2010.

[47] A. da Candelaria Renesto, M. Sartori, and M. Campos, "Crosslinking and intrastromal corneal ring segment," Arquivos Brasileiros de Oftalmologia, vol. 74, no. 1, pp. 67-74, 2011.

[48] G. D. Kymionis, V. F. Diakonis, M. Kalyvianaki et al., "One-year follow-up of corneal confocal microscopy after corneal crosslinking in patients with post laser in situ keratosmileusis ectasia and keratoconus," American Journal of Ophthalmology, vol. 147, no. 5, pp. 774.e1-778.e1, 2009.

[49] D. Asri, D. Touboul, P. Fournié et al., "Corneal collagen crosslinking in progressive keratoconus: Multicenter results from the French National Reference Center for Keratoconus," Journal of Cataract and Refractive Surgery, vol. 37, no. 12, pp. 2137-2143, 2011.

[50] B. J. Dahl, E. Spotts, and J. Q. Truong, "Corneal collagen crosslinking: an introduction and literature review," Optometry, vol. 83, no. 1, pp. 33-42, 2012.

[51] F. Hafezi, J. Kanellopoulos, R. Wiltfang, and T. Seiler, "Corneal collagen crosslinking with riboflavin and ultraviolet A to treat induced keratectasia after laser in situ keratomileusis," Journal of Cataract and Refractive Surgery, vol. 33, no. 12, pp. 2035-2040, 2007.

[52] P. S. Hersh, S. A. Greenstein, and K. L. Fry, "Corneal collagen crosslinking for keratoconus and corneal ectasia: one-year results," Journal of Cataract and Refractive Surgery, vol. 37, no. 1, pp. 149-160, 2011.

[53] M. Hovakimyan, R. F. Guthoff, and O. Stachs, "Collagen cross-linking: current status and future directions," Journal of Ophthalmology, vol. 2012, Article ID 406850, 12 pages, 2012.

[54] P. Vinciguerra, F. I. Camesasca, E. Albè, and S. Trazza, "Corneal collagen cross-linking for ectasia after excimer laser refractive surgery: 1-year results," Journal of Refractive Surgery, vol. 26, no. 7, pp. 486-497, 2010.

[55] C. Mazzotta, C. Traversi, S. Baiocchi et al., "Corneal healing after riboflavin ultraviolet-A collagen cross-linking determined by confocal laser scanning microscopy in vivo: early and late modifications," American Journal of Ophthalmology, vol. 146, no. 4, pp. 527-533, 2008.

[56] T. Koller, M. Mrochen, and T. Seiler, "Complication and failure rates after corneal crosslinking," Journal of Cataract and Refractive Surgery, vol. 35, no. 8, pp. 1358-1362, 2009.

[57] T. Koller, B. Pajic, P. Vinciguerra, and T. Seiler, "Flattening of the cornea after collagen crosslinking for keratoconus," Journal of Cataract and Refractive Surgery, vol. 37, no. 8, pp. 1488-1492, 2011.

[58] T. Seiler and F. Hafezi, "Corneal cross-linking-induced stromal demarcation line," Cornea, vol. 25, no. 9, pp. 1057-1059, 2006. 
[59] Z. Dong and X. Zhou, "Collagen cross-linking with riboflavin in a femtosecond lasercreated pocket in rabbit corneas: 6-month results," The American Journal of Ophthalmology, vol. 152, no. 1, pp. 22-27, 2011.

[60] J. Beckman Rehnman, C. C. Janbaz, A. Behndig, and C. Lindén, "Spatial distribution of corneal light scattering after corneal collagen crosslinking," Journal of Cataract and Refractive Surgery, vol. 37, no. 11, pp. 1939-1944, 2011.

[61] D. S. Grewal, G. S. Brar, R. Jain, V. Sood, M. Singla, and S. P. S. Grewal, "Corneal collagen crosslinking using riboflavin and ultraviolet-A light for keratoconus: one-year analysis using Scheimpflug imaging," Journal of Cataract and Refractive Surgery, vol. 35, no. 3, pp. 425-432, 2009.

[62] T. Koller, H. P. Iseli, F. Hafezi, P. Vinciguerra, and T. Seiler, "Scheimpflug imaging of corneas after collagen cross-linking," Cornea, vol. 28, no. 5, pp. 510-515, 2009.

[63] M. Doors, N. G. Tahzib, F. A. Eggink, T. T. J. M. Berendschot, C. A. B. Webers, and R. M. M. A. Nuijts, "Use of anterior segment optical coherence tomography to study corneal changes after collagen cross-linking," The American Journal of Ophthalmology, vol. 148, no. 6, pp. 844.e2-851.e2, 2009.

[64] E. Spoerl, M. Mrochen, D. Sliney, S. Trokel, and T. Seiler, "Safety of UVA-riboflavin cross-linking of the cornea," Cornea, vol. 26, no. 4, pp. 385-389, 2007.

[65] S. A. Greenstein, V. P. Shah, K. L. Fry, and P. S. Hersh, "Corneal thickness changes after corneal collagen crosslinking for keratoconus and corneal ectasia: one-year results," Journal of Cataract and Refractive Surgery, vol. 37, no. 4, pp. 691-700, 2011.

[66] D. A. Luce, "Determining in vivo biomechanical properties of the cornea with an ocular response analyzer," Journal of Cataract and Refractive Surgery, vol. 31, no. 1, pp. 156-162, 2005.

[67] W. J. Dupps Jr. and S. E. Wilson, "Biomechanics and wound healing in the cornea," Experimental Eye Research, vol. 83, no. 4, pp. 709-720, 2006.

[68] W. J. Dupps Jr., "Biomechanical modeling of corneal ectasia," Journal of Refractive Surgery, vol. 21, no. 5, pp. 186-190, 2005.

[69] W. J. Dupps Jr., "Hysteresis: new mechanospeak for the ophthalmologist," Journal of Cataract and Refractive Surgery, vol. 33, no. 9, pp. 1499-1501, 2007.

[70] C. W. McMonnies, "Assessing corneal hysteresis using the ocular response analyzer," Optometry and Vision Science, vol. 89, no. 3, pp. E343-E349, 2012.

[71] Y. Goldich, Y. Barkana, Y. Morad, M. Hartstein, I. Avni, and D. Zadok, "Can we measure corneal biomechanical changes after collagen cross-linking in eyes with keratoconus? A pilot study," Cornea, vol. 28, no. 5, pp. 498-502, 2009.

[72] E. Spoerl, N. Terai, F. Scholz, F. Raiskup, and L. E. Pillunat, "Detection of biomechanical changes after corneal crosslinking using ocular response analyzer software," Journal of Refractive Surgery, vol. 27, no. 6, pp. 452-457, 2011.

[73] P. Vinciguerra, E. Albè, A. M. Mahmoud, S. Trazza, F. Hafezi, and C. J. Roberts, "Intra- and postoperative variation in ocular response analyzer parameters in keratoconic eyes after corneal cross-linking," Journal of Refractive Surgery, vol. 26, no. 9, pp. 669-676, 2010.

[74] D. H. Glass, C. J. Roberts, A. S. Litsky, and P. A. Weber, "A viscoelastic biomechanical model of the cornea describing the effect of viscosity and elasticity on hysteresis," Investigative Ophthalmology and Visual Science, vol. 49, no. 9, pp. 3919-3926, 2008.
[75] R. Ambrósio Jr., L. P. Nogueira, D. L. Caldas et al., "Evaluation of corneal shape and biomechanics before LASIK," International Ophthalmology Clinics, vol. 51, no. 2, pp. 11-38, 2011.

[76] M. Mikielewicz, K. Kotliar, R. I. Barraquer, and R. Michael, "Airpulse corneal applanation signal curve parameters for the characterisation of keratoconus," British Journal of Ophthalmology, vol. 95, no. 6, pp. 793-798, 2011.

[77] R. Ambrósio Jr., I. Ramos, A. Luz et al., "Dynamic ultra high speed scheimpflug imaging for assessing corneal biomechanical properties," Revista Brasileira de Oftalmologia, vol. 72, no. 2, pp. 99-102, 2013.

[78] C. Mazzotta, T. Caporossi, R. Denaro et al., "Morphological and functional correlations in riboflavin UV A corneal collagen cross-linking for keratoconus," Acta Ophthalmologica, vol. 90, no. 3, pp. 259-265, 2012.

[79] D. Touboul, N. Efron, D. Smadja, D. Praud, F. Malet, and J. Colin, "Corneal confocal microscopy following conventional, transepithelial, and accelerated corneal collagen cross-linking procedures for keratoconus," Journal of Refractive Surgery, vol. 28, no. 11, pp. 769-775, 2012.

[80] J. S. Dhaliwal and S. C. Kaufman, "Corneal collagen crosslinking: a confocal, electron, and light microscopy study of eye bank corneas," Cornea, vol. 28, no. 1, pp. 62-67, 2009.

[81] G. D. Kymionis, V. F. Diakonis, M. Kalyvianaki et al., "One-year follow-up of corneal confocal microscopy after corneal crosslinking in patients with post laser in situ Keratosmileusis ectasia and Keratoconus," American Journal of Ophthalmology, vol. 147, no. 5, pp. 774-778, 2009.

[82] K. Samaras, D. P. O’Brart, J. Doutch, S. Hayes, J. Marshall, and K. M. Meek, "Effect of epithelial retention and removal on ribofl avin absorption in porcine corneas," Journal of Refractive Surgery, vol. 25, no. 9, pp. 771-775, 2009.

[83] T. A. Alhamad, D. P. S. O’Brart, N. A. L. O’Brart, and K. M. Meek, "Evaluation of transepithelial stromal riboflavin absorption with enhanced riboflavin solution using spectrophotometry," Journal of Cataract and Refractive Surgery, vol. 38, no. 5, pp. 884-889, 2012.

[84] K. M. Bottos, A. G. Oliveira, P. A. Bersanetti et al., "Corneal absorption of a new riboflavin-nanostructured system for transepithelial collagen cross-linking," PLoS ONE, vol. 8, no. 6, Article ID e66408, 2013.

[85] G. Bikbova and M. Bikbov, "Transepithelial corneal collagen cross-linking by iontophoresis of riboflavin," Acta Ophthalmologica, vol. 92, no. 1, pp. e30-e34, 2014.

[86] A. J. Kanellopoulos and P. S. Binder, "Management of corneal ectasia after LASIK with combined, same-day, topographyguided partial transepithelial PRK and collagen cross-linking: the Athens protocol," Journal of Refractive Surgery, vol. 27, no. 5, pp. 323-331, 2011.

[87] R. Bunsen and H. E. Roscoe, "Photochemical researches, part V: on the measurement of the chemical action of direct and diffuse sunlight," Proceedings of the Royal Society of London, vol. 12, pp. 306-312, 1862.

[88] N. E. K. Cartwright, J. R. Tyrer, and J. Marshall, "Age-related differences in the elasticity of the human cornea," Investigative Ophthalmology and Visual Science, vol. 52, no. 7, pp. 4324-4329, 2011.

[89] P. D. Jaycock, L. Lobo, J. Ibrahim, J. Tyrer, and J. Marshall, "Interferometric technique to measure biomechanical changes in the cornea induced by refractive surgery," Journal of Cataract and Refractive Surgery, vol. 31, no. 1, pp. 175-184, 2005. 
[90] G. Scarcelli, R. Pineda, and S. H. Yun, "Brillouin optical microscopy for corneal biomechanics," Investigative Ophthalmology and Visual Science, vol. 53, no. 1, pp. 185-190, 2012.

[91] G. Grabner, R. Eilmsteiner, C. Steindl, J. Ruckhofer, R. Mattioli, and W. Husinsky, "Dynamic corneal imaging," Journal of Cataract and Refractive Surgery, vol. 31, no. 1, pp. 163-174, 2005.

[92] J. A. Bonatti, S. J. Bechara, P. C. Carricondo, and N. KaraJosé, "Proposal for a new approach to corneal biomechanics: dynamic corneal topography," Arquivos Brasileiros de Oftalmologia, vol. 72, no. 2, pp. 264-267, 2009. 


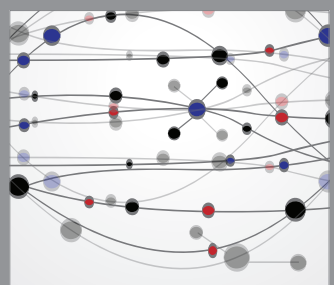

The Scientific World Journal
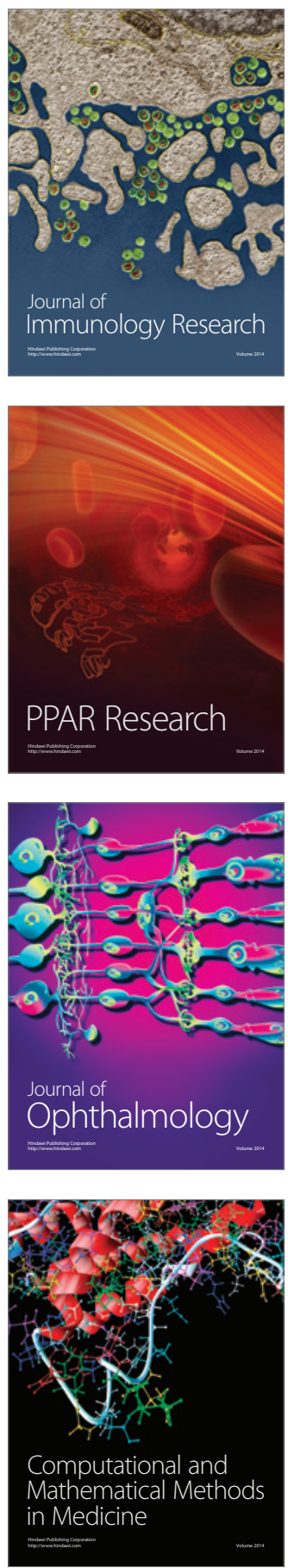

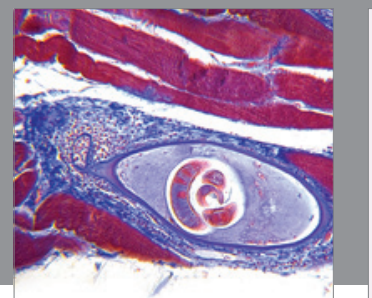

Gastroenterology

Research and Practice
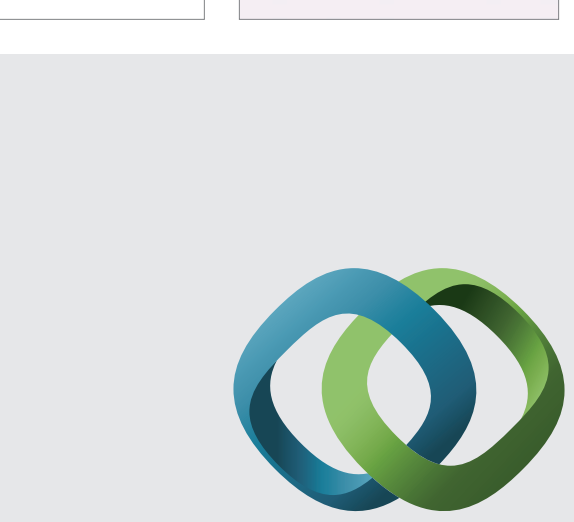

\section{Hindawi}

Submit your manuscripts at

http://www.hindawi.com
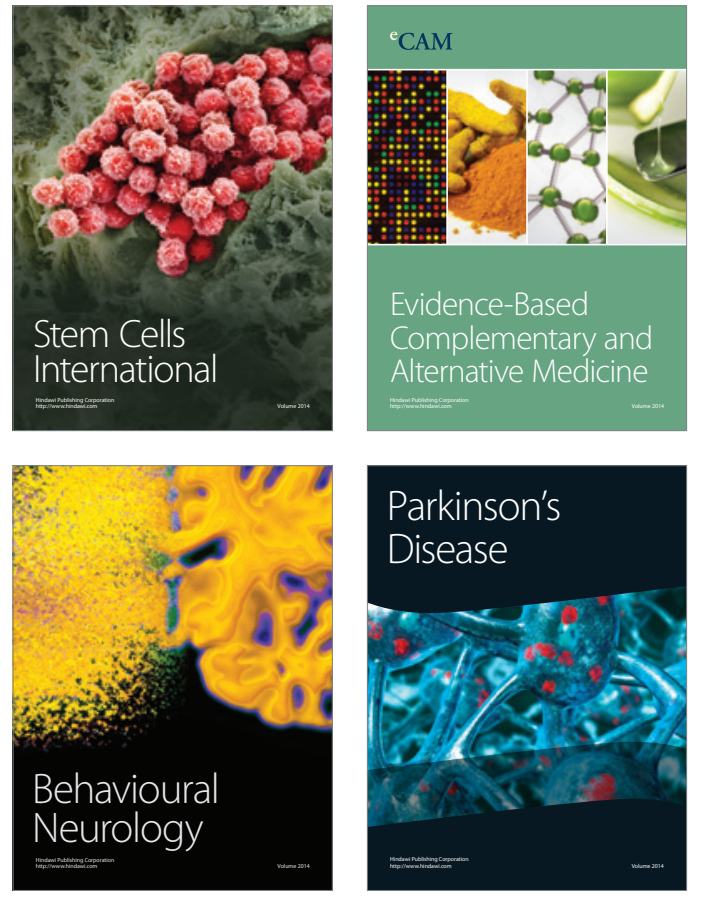
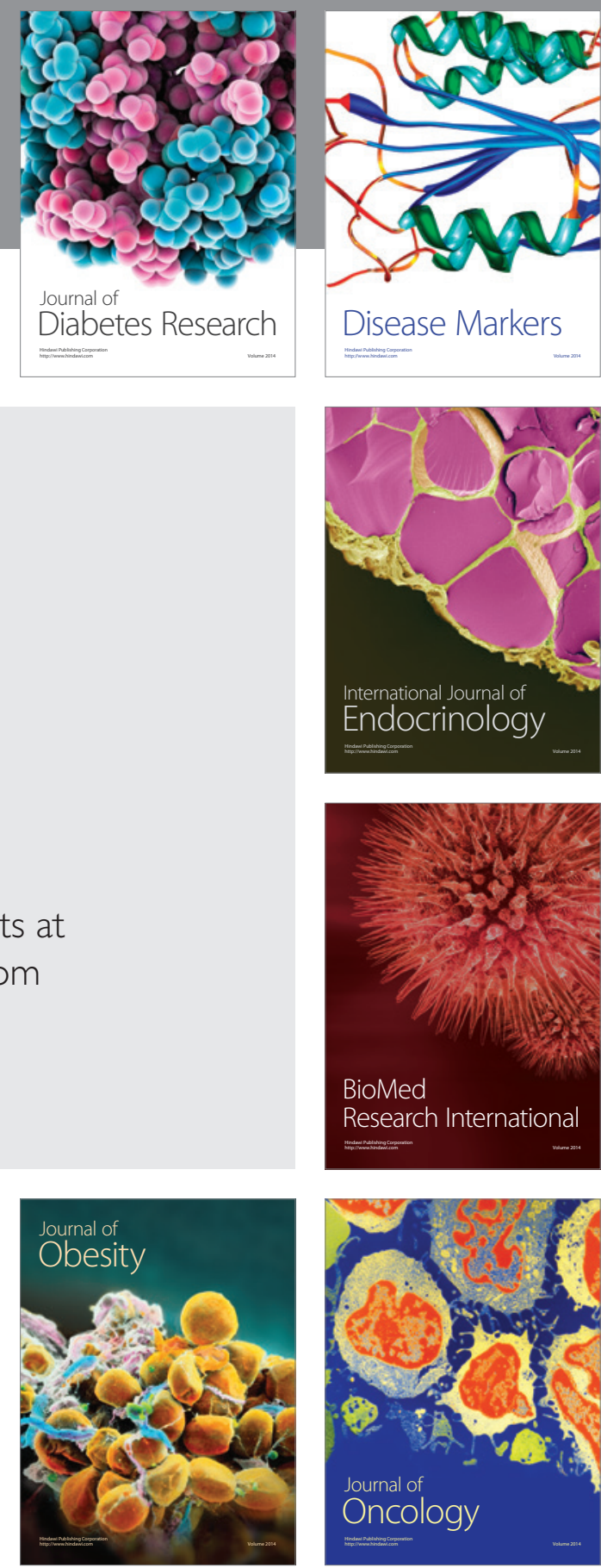

Disease Markers
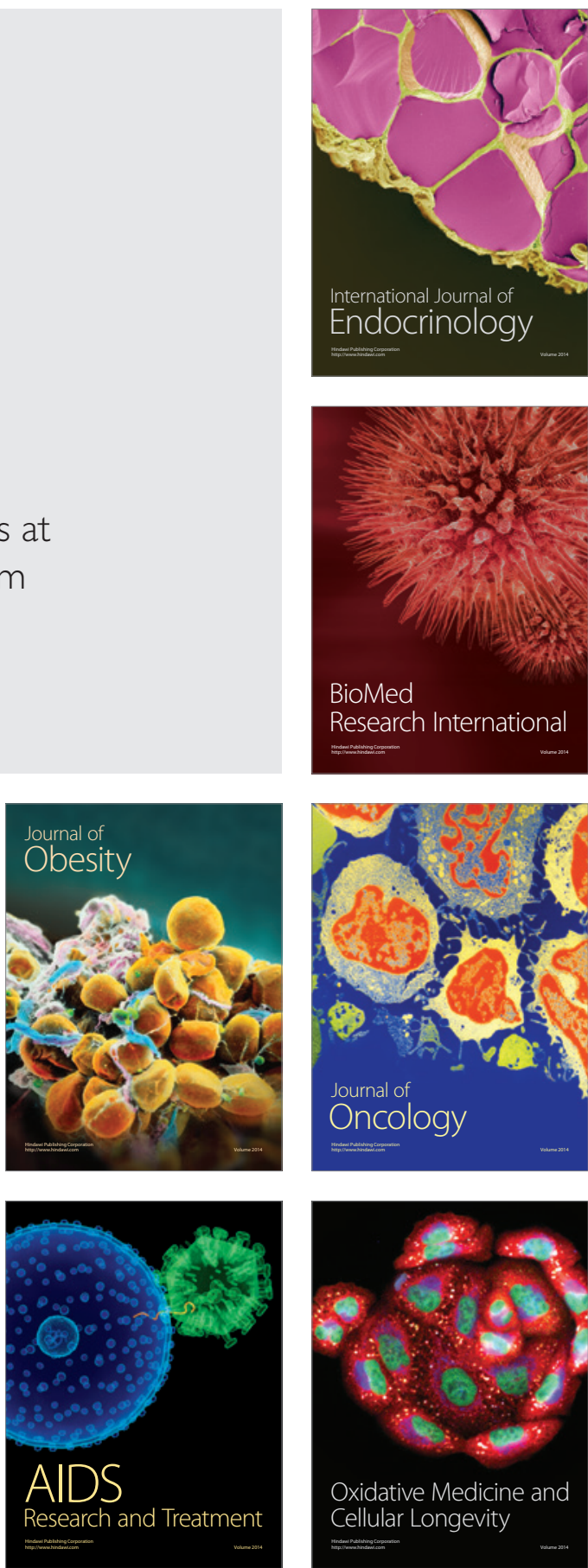